\title{
Protogalactic Extension of the Parker Bound
}

\author{
M. J. Lewis, Katherine Freeseł, and Gregory Tarle ${ }^{\ddagger}$ \\ Randall Physics Laboratory \\ University of Michigan \\ Ann Arbor, MI 48109-1120
}

June 20, 2021

\begin{abstract}
We extend the Parker bound on the galactic flux $\mathcal{F}$ of magnetic monopoles. By requiring that a small initial seed field must survive the collapse of the protogalaxy, before any regenerative dynamo effects become significant, we develop a stronger bound. The survival and continued growth of an initial galactic seed field $\leq 10^{-9} \mathrm{G}$ demand that $\mathcal{F} \leq 5 \times 10^{-21}\left(\mathrm{~m} / 10^{17} \mathrm{GeV}\right) \mathrm{cm}^{-2} \mathrm{sec}^{-1} \mathrm{sr}^{-1}$. For a given monopole mass, this bound is four and a half orders of magnitude more stringent than the previous 'extended Parker bound' [1], but is more speculative as it depends on assumptions about the behavior of magnetic fields during protogalactic collapse. For monopoles which do not overclose the Universe $\left(\Omega_{m}<1\right)$, the maximum flux allowed is now $8 \times 10^{-19}$ $\mathrm{cm}^{-2} \mathrm{~s}^{-1} \mathrm{sr}^{-1}$, a factor of 150 lower than the maximum flux allowed by the extended Parker bound.
\end{abstract}

\section{Introduction}

The existence of magnetic monopoles has long been an intriguing prospect, motivating both theorists and experimentalists for more than 50 years. Dirac [2] first showed that magnetic monopoles could be accomodated within electromagnetic theory if their magnetic charge, $g$, is given by an integer multiple of $\hbar c / 2 e$. In 1974, t'Hooft [3] and Polyakov [4] independently demonstrated that monopoles are necessary components of any Grand Unified

\footnotetext{
*Electronic mail: lewism@umich.edu

${ }^{\dagger}$ Electronic mail: ktfreese@umich.edu

${ }^{\ddagger}$ Electronic mail: tarle@umalp1.physics.1sa.umich.edu
} 
theory (GUT) that includes electromagnetism. If GUTs are shown to be correct, monopoles with mass $m \sim m_{G U T} / \alpha_{G U T} \sim 10^{15}-10^{19} \mathrm{GeV}$ are expected. However, no magnetic monopole has ever been observed, and the cosmic abundance of monopoles remains an open question.

The experimental search for magnetic monopoles has intensified in the past decade. The MACRO experiment [5], now fully operational, has placed stringent limits (90\% confidence level) on the terrestrial monopole flux. For a combined direct magnetic monopole search using scintillators, streamer tubes, and nuclear track detectors, MACRO has reached flux sensitivities below $3.2 \times 10^{-16} \mathrm{~cm}^{-2} \mathrm{sec}^{-1} \mathrm{sr}^{-1}$ for monopoles in the velocity range $10^{-4}<\beta<10^{-1}$.

Estimates of monopole abundance based on GUT phase transitions yield a monopole density which overcloses the universe by several orders of magnitude [6]. Inflationary models [7] can resolve this "monopole problem" by reducing the monopole abundance within the observable universe to an exponentially small value. It is difficult to subsequently predict theoretical expectations for the number of monopoles in the universe. Hence astrophysics can provide useful benchmark abundances for experimenters looking for monopoles, and the most practical constraints on the present day magnetic monopole flux arise from these astrophysical bounds, which fall into three categories: 1) cosmological bounds, which require that monopoles can provide at most the critical density of the universe; 2) nucleon decay catalysis bounds, arising from the hypothesis that monopoles catalyze nucleon decay in the cores of neutron stars and white dwarfs; and, 3) Parker-type bounds, which demand that monopoles not drain energy from astrophysical magnetic fields faster than they are regenerated. Catalysis bounds are by far the most stringent [8], but depend on the value of the cross-section for monopole catalyzed nucleon decay. In this paper, we reconsider the Parkertype bounds, noting that a stronger limit may be derived by considering the survival of a magnetic seed field during the collapse of the protogalaxy.

Parker first emphasized that the existence of observable Galactic magnetic fields must place an upper limit on the magnetic monopole flux [9]. The presence of a Galactic magnetic field indicates a relative dearth of magnetic monopoles.

The original flux bound based upon the survival of today's galactic magnetic field is the most straightforward [9]. Magnetic monopoles which move along field lines absorb kinetic energy at the expense of the field. In order for the galactic field to survive, the magnetic monopoles must not drain field energy faster than it is regenerated by the dynamo, presumed to act on the 
order of the Galactic rotation time period, $10^{8}$ yrs [10]. This implies an upper bound on the flux of monopoles, $\mathcal{F} \leq 10^{-16} \mathrm{~cm}^{-2} \mathrm{sec}^{-1} \mathrm{sr}^{-1}$. This bound was later shown to be mass dependent [11]. Subsequently Adams et al [1] obtained an "extended Parker bound" by requiring survival and growth of a small galactic seed field after the collapse of the protogalaxy:

$$
\mathcal{F} \leq 10^{-16}\left(\mathrm{~m} / 10^{17} \mathrm{GeV}\right) \mathrm{cm}^{-2} \mathrm{~s}^{-1} \mathrm{sr}^{-1}
$$

Here we extend these bounds further by considering an even earlier epoch in the history of the Galaxy, namely during collapse of the protogalaxy to the size that it has today. The flux bounds that we obtain in this way are the most stringent Parker type bounds to date for three reasons: (i) the magnetic field at this early time is very small with a larger coherence length than today, (ii) the protogalaxy starts to collapse at a higher redshift $z \sim 5$, and (iii) at this early time no dynamo can yet be important, so that we take the primary enhancement mechanism for the B field to be flux freezing, $\frac{d}{d t}\left(B R^{2}\right)=0$. Section 2 presents the protogalactic extension of the Parker bound. Section 3 concludes with a discussion of caveats to this bound, in particular the suggestion by Kulsrud et al [12] that Kolmogorov turbulence amplifies the magnetic field. The farther back one goes in the history of the Galaxy, the more speculative the bounds become, since we do not really understand the origin of the galactic magnetic field.

\section{The Protogalactic Extension}

The origin of the Galactic magnetic field remains an outstanding problem of theoretical astrophysics. Faraday rotation measurements of polarized pulsar radio emissions indicate a mean Galactic magnetic field strength of $(2-3) \times 10^{-6} \mathrm{G}$. The polarization of starlight by aligned interstellar dust grains suggests a large scale field, coherent over scales of (1-2) kpc, and extending more or less in the azimuthal direction in the disk of the Galaxy. Such fields may have originated from a large (i.e. $\sim 10^{-9} \mathrm{G}$ ) primordial seed field amplified by the collapse of the Galaxy, or by a much smaller seed field $\left(\sim 10^{-20} \mathrm{G}\right)$ that was subsequently amplified by a fast dynamo. Recent work by Enqvist 13] has argued that a large scale $10^{-20} \mathrm{G}$ seed field can arise via turbulent evolution from microscopic primordial magnetic fields such as those arising during cosmological phase transitions. Additional schemes for obtaining seed fields by considering magnetic flux entrained in the winds from young stellar objects [14], or by various battery mechanisms 
[16] also exist, and under the most favorable conditions can produce seed fields of $10^{-11} \mathrm{G}$. As an alternative to dynamo amplification of a small seed field, Kulsrud et. al. [12] argue for a protogalactic origin for the magnetic field, wherein primary amplification of the field comes from Kolmogorov turbulence; we will further consider this possibility below. The protogalactic extension to the flux bound is based on the fact that, regardless of which scenario is correct, the existence of a field today requires that some field must exist after the collapse of the protogalaxy. To be conservative, we will take the largest possible protogalactic field value, $B_{0}=10^{-9} \mathrm{G}$, which assumes that flux freezing was the only early amplification mechanism. This value is conservative in that it will give the least restrictive bound on the monopole flux.

Clearly the Galactic magnetic field is most vulnerable to dissipation in the absence of a regenerating dynamo. By considering the evolution of a small seed field in an era during which such a dynamo is not yet functioning, or when its effect is negligible (i.e., the protogalactic era), we develop a tighter bound; that is, we require a smaller upper bound on the flux of monopoles to avoid extinguishing the existing Galactic field. The time evolution of the magnetic field in the protogalaxy is governed by competition between amplification due to flux freezing, and dissipation due to a possible flux of magnetic monopoles. The details of this competition may be modeled by an equation of motion for the magnetic field of the form [1].

$$
\frac{d B}{d t}=\gamma_{\text {coll }} B-\frac{g \mathcal{F}}{1+\mu / B}
$$

Here the first term on the right hand side describes the field amplification due to flux freezing and the second term describes dissipation by a flux of monopoles. Each of the quantities has been written in non-dimensional units. Here $g$ is the magnetic charge in Dirac units (we take $g=1$ ). The magnetic field is measured in units of the present day Galactic field strength, $3 \times 10^{-6} G$. The parameter $\gamma_{\text {coll }}$ represents the growth rate of the galactic field, and has units of $10^{-8} \mathrm{yr}^{-1}$ (i.e. the Galactic rotation rate). The flux, $\mathcal{F}$, is measured in units of $1.2 \times 10^{-16} \mathrm{~cm}^{-2} \mathrm{sec}^{-1} \mathrm{sr}^{-1}$. Finally, the dissipation term depends upon $\mu$, where $\mu=m v^{2} / \ell ; m$ is the monopole mass in units of $10^{17} \mathrm{GeV}, v$ is the monopole velocity in units of $10^{-3} c$, and $\ell$ is the coherence length of the Galactic field measured in units of $1 \mathrm{kpc}$ [1, 11].

As the protogalaxy collapses, we expect that the only significant amplification mechanism will be flux freezing. This results in an effective growth 
rate, $\gamma_{\text {coll }} \approx 2 / \tau_{\text {coll }}$, where $\tau_{\text {coll }}$ is the collapse time of the galaxy, approximately $10^{9}$ yrs [15]. In the dimensionless units just defined, $\gamma_{\text {coll }}=0.2$. Dynamo action during this era is negligible since, prior to collapse, the rotation period is extremely large compared to the collapse time. The rotation period today is $10^{8} \mathrm{yr}$; using conservation of angular momentum, we see that during the protogalactic epoch, when the radius was larger by a factor of roughly 50 , the rotation period was $>10^{11} \mathrm{yr}$. In comparison, as mentioned above, the collapse timescale is roughly $10^{9} \mathrm{yr}$. Consequently, our results are independent of any specific dynamo model. Additional dissipative effects which depend upon the magnitude of the Galactic magnetic field, such as turbulent dissipation (which evolves as $B^{2}$ ), may also be ignored.

One point of concern is the value of the coherence length of the Galactic field during the protogalactic era, as the dissipative term in equation (1) depends upon $\mu=m v^{2} / \ell g$. Before Galactic collapse, we expect this coherence length to be much longer than today's value of $\sim 1 \mathrm{kpc}$. Although the value is highly uncertain, we expect $l \sim 30-100 \mathrm{kpc}$; hence, throughout the paper we adopt the value $\ell=50 \mathrm{kpc}$.

The monopole velocity $v$ is in units of $10^{-3} \mathrm{c}$, as mentioned above. We expect a massive monopole to acquire this velocity due to gravitational acceleration by the Galaxy during infall. In a model of galaxy formation in which the dark matter aggregates first into a dark halo, and the baryons subsequently fall into the potential well provided by the dark matter, it is reasonable to assume that the monopoles attained the virial velocity of $10^{-3} \mathrm{c}$ once the dark matter haloes came into existence. Subequently, the protogalactic collapse of the baryons drags the magnetic field with it. If some other model of galaxy formation is considered in which the dark haloes do not form first, then it is possible that the monopoles were moving somewhat slower; a slower monopole velocity will only lead to a tighter bound on the monopole flux. Hence we will use the value $10^{-3}$ as the lowest monopole velocity, in order to be on the conservative side.

Light monopoles $\left(\mu<<B_{0}\right)$ were accelerated to higher velocities by the galactic field, while 'heavy' monopoles $\left(\mu>>B_{0}\right)$ did not have their velocities changed significantly. For our fiducial values of $B_{0} \sim 10^{-9} \mathrm{G}$ and $\ell=50 \mathrm{kpc}$, we expect that monopoles heavier than $2 \times 10^{15} \mathrm{GeV}$ were moving at $10^{-3}$ while lighter monopoles were moving faster. As seen in figure 1 and discussed further below, the monopole flux bound changes slope at this value of the mass: the bound is linear as a function of mass for monopoles heavier than $2 \times 10^{15} \mathrm{GeV}$ and flat for lower masses.

Given equation (2), the possible behavior of the field is straightforward 
to determine. If the field is to survive, we must have $d B / d t>0$. This requires

$$
\gamma_{\text {coll }} B+\left(\gamma_{\text {coll }} \mu-g \mathcal{F}\right)>0
$$

If the monopole flux $\mathcal{F}<\mu \gamma_{\text {coll }}$, then the field survives for all initial values of the field strength. This behavior holds for $\mu>B_{0}$, i.e., for heavy monopoles with $m>2 \times 10^{15}\left(\frac{B_{0}}{10^{-9} \mathrm{G}}\right) \mathrm{GeV}$, and gives rise to the linear dependence of the monopole flux bound on the mass (see the Figure). If, on the other hand, $\mathcal{F}>\mu \gamma_{\text {coll }}$, then only initial field strengths $B_{0}>B_{c} \equiv \mathcal{F} / \gamma_{\text {coll }}-\mu$ will survive. This behavior holds for $\mu<B_{0}$, i.e., for light monopoles with $m<2 \times 10^{15}\left(\frac{B_{0}}{10^{-9} \mathrm{G}}\right) \mathrm{GeV}$, and gives rise to the flat part of the monopole flux bound as a function of mass (see the Figure). Thus, for the protogalactic field $B_{0}$ to survive during collapse, the flux of monopoles at this time must obey one of two bounds

$$
\begin{gathered}
\mathcal{F}<\mu \gamma_{\text {coll }} \quad \text { or } \\
\mathcal{F}<\left(B_{0}+\mu\right) \gamma_{\text {coll }} .
\end{gathered}
$$

We note that the flux of monopoles is $\mathcal{F}=n v /(4 \pi)$, where $n$ is the monopole number density, which scales with the redshift as $n \sim(1+z)^{3}$. The flux today is smaller than the flux at the time of protogalactic collapse by a factor of $\left(1+z_{\text {proto }}\right)^{3} \approx 100$, where $z_{\text {proto }}$ is the redshift of galaxy formation. Hence the flux of monopoles today is constrained to be smaller than the bounds of eqns. (4) and (5) by this factor.

Thus we obtain an analytical estimate for the bound on monopole flux,

$$
\mathcal{F}<5 \times 10^{-21}\left(\frac{m}{10^{17} \mathrm{GeV}}\right) \mathrm{cm}^{-2} \mathrm{sec}^{-1} \mathrm{sr}^{-1}
$$

for $m>2 \times 10^{15} \mathrm{GeV}$, and

$$
\mathcal{F}<9 \times 10^{-23}\left(\frac{B_{0}}{10^{-9} \mathrm{G}}\right) \mathrm{cm}^{-2} \sec ^{-1} \mathrm{sr}^{-1}
$$

for $m<2 \times 10^{15} \mathrm{GeV}$.

\section{Discussion and Conclusion}

Without the support of a regenerative mechanism, the Galactic magnetic field is particularly susceptible to dissipation by a flux of magnetic monopoles. 
We have exploited this vulnerability of a small B field early in the history of the Galaxy to arrive at strict bounds on the monopole flux given in eqns. (6) and (7). Figure 1 summarizes the new flux bounds, displays previous Parker bounds, and has a line indicating a closure density of monopoles. Here, $\Omega_{m}=\rho_{m} / \rho_{c}$ is the mass density of monopoles $\rho_{m}$ in units of the critical density required to close the universe, $\rho_{c}=2.8 \times 10^{-29} \mathrm{~g} \mathrm{~cm}^{-3}$ (for Hubble constant $H_{o}=70 \mathrm{~km} \mathrm{~s}^{-1} \mathrm{Mpc}^{-1}$ ).

Note that the new protogalactic extension of the Parker bound differs from the extended Parker bound even at low monopole masses, where the curves flatten out. The reason for this discrepancy is twofold: i) the collapse timescale in eqn. (2) is $1 / \gamma_{\text {coll }} \sim 10^{9} \mathrm{yr}$, whereas the corresponding rotation timescale in the extended Parker bound is $200 \mathrm{Myr}$; ii) we have taken the coherence length of the magnetic field in the protogalaxy to be $50 \mathrm{kpc}$, whereas the coherence length in the Galaxy is roughly $1 \mathrm{kpc}$.

As a caveat, note that the bounds in this paper rest on the assumption that no amplifying mechanism other than flux freezing exists during collapse. Recent work 12] suggests that a preglactic seed field, amplified by a postcollapse dynamo may not be the only way to generate the observed field. Kulsrud et al. suggest the possibility that Kolmogorov turbulence and flux freezing together, acting on a small seed field can produce the observed Galactic field, without recourse to a large scale dynamo. In this case, the equation of motion becomes

$$
\frac{d B}{d t}=\left(\gamma_{\text {coll }}+\gamma_{\text {turb }}\right) B-\frac{g \mathcal{F}}{1+\mu / B}
$$

where the $\gamma_{\text {turb }}$ factor measures field growth due to Kolmogorov turbulence, and $\gamma_{\text {turb }} \sim 600\left(\rho_{B} / \rho_{D}\right)$ in the previously defined units; here, $\rho_{B} / \rho_{D}$ is the ratio of baryonic matter to dark matter. Hence, looking at eqns (4) and (5) one might conclude that in this scenario the flux bounds would be weaker by at least this factor. However, there is another more important difference between the scenario of Kulsrud et al. and the flux freezing we have described above: in the Kulsrud et al. model, the coherence length of the magnetic field during this era is much smaller, on the order of viscous length scales. If indeed $\ell$ is this small, then eqns. (5) and (6) do not result in an interesting bound. Monopoles would only begin to dominate the contribution to $d B / d t$ once $\ell$ becomes large; currently, in this model, it is unclear when this would happen. However, numerical simulations employed in 12 lack the resolution to demonstrate that homogenous Kolmogorov turbulence actually occurs down to the smallest scales, so the final word on 
this issue must wait until alternate theoretical methods are developed or computational power is increased.

Modulo this caveat, we have found a protogalactic extension to the Parker bound that is four and a half orders of magnitude more stringent than the extended Parker bound [1] for a given monopole mass.

Monopoles with $\Omega_{m}<1$ that satisfy the extended Parker bound of Ref. [1] were in principle accessible to direct searches (that rely on electromagnetic interactions of monopoles) in an experimental apparatus like MACRO with longer running time. However, monopoles with $\Omega_{m}<1$ that satisfy the new protogalactic extension of the Parker bound presented in this paper are no longer accessible to existing direct search experiments. The new maximum monopole flux at the closure bound is $\mathcal{F}=8 \times 10^{-19} \mathrm{~cm}^{-2} \mathrm{~s}^{-1} \mathrm{sr}^{-1}$ and occurs for monopole mass near the Planck scale $\sim 10^{19} \mathrm{GeV}$. This maximum flux at the closure bound is a factor of 150 lower than the maximum allowed by the extended Parker bound, which occurs for a monopole mass near the GUT scale $\sim 10^{17} \mathrm{GeV}$. Thus MACRO would have to be scaled up in size by a factor of more than 100 to access the new maximum flux compatible with the protogalactic extension as well as the closure bound. Hence the new monopole bound in this paper, while more uncertain than previous bounds, would have serious consequences for experimental searches.

\section{Figure Caption}

Monopole flux limits as a function of the monopole mass $m$ in GeV. The line labeled TPB Bound shows the modified Parker bound obtained in Ref. [11]. The dotted lines show the extended Parker bound of Ref. [1]. The solid lines show the protogalactic extension of the Parker bound of this paper. The line labeled $\Omega_{M}$ represents the bound obtained by assuming monopoles are uniformly distributed throughout the Universe but do not 'over close' the Universe. Here, we have taken $H_{o}=70 \mathrm{~km} \mathrm{~s}^{-1} \mathrm{Mpc}^{-1}$. If the monopoles are clustered with galaxies, this closure bound becomes weaker by a factor of $10^{5}$.

\section{Acknowledgements}

We would like to thank the Department of Energy for support at the University of Michigan. K. Freese would like to thank the Max Planck Institut fuer Physik in Muenchen, where some of this work was completed, for hospitality during her stay. We also thank F. Adams, G. Laughlin, and M. Turner for useful conversations. 


\section{References}

[1] F. Adams, et. al. Phys. Rev. Lett. 702511 (1993)

[2] P.A.M. Dirac, Phys. Rev. 74, 817 (1948)

[3] G. t'Hooft, Nucl. Phys. B79, 276 (1974)

[4] A. Polyakov, JETP Letters 20, 194 (1974)

[5] B.C. Choudhary et al, in Proc. of the 26th international Cosmic Ray Conference, Salt Lake City, Utah, 2, 332 (1999)

[6] T.W.B. Kibble, J. Phys. A9, 1387 (1976)

[7] A.H. Guth, Phys. Rev., D23, 347 (1981)

[8] E.W. Kolb, S. Colgate, and J.A. Harvey, Phys. Rev. Lett., 49, 1373 (1982); S. Dimopoulos, J. Preskill, and F. Wilczek, Phys. Lett., 119B, 320 (1982); K. Freese, M.S. Turner, and D.N. Schramm, Phys. Rev. Lett., 51, 1625 (1983); K. Freese, Ap. J., 286, 216 (1984); J.A. Frieman, K. Freese, and M.S. Turner, Ap. J., 335, 844 (1988).

[9] E.N. Parker, Astrophys. J. 160, 383 (1970)

[10] E.N. Parker, Astrophys. J. 163, 255 (1971)

[11] M.S. Turner, E.N. Parker, and T.J. Bogdan, Phys. Rev. D26, 1296 (1982)

[12] R.M. Kulsrud, et. al., Astroph. J. 480, 480 (1997)

[13] K. Enqvist, Preprint astro-ph/9803196 (1999)

[14] E.T. Vishniac, Preprint (1992)

[15] J. Silk, Nature 351, 191 (1991).

[16] L. Biermann, Naturforsch 5a 65 (1950) 


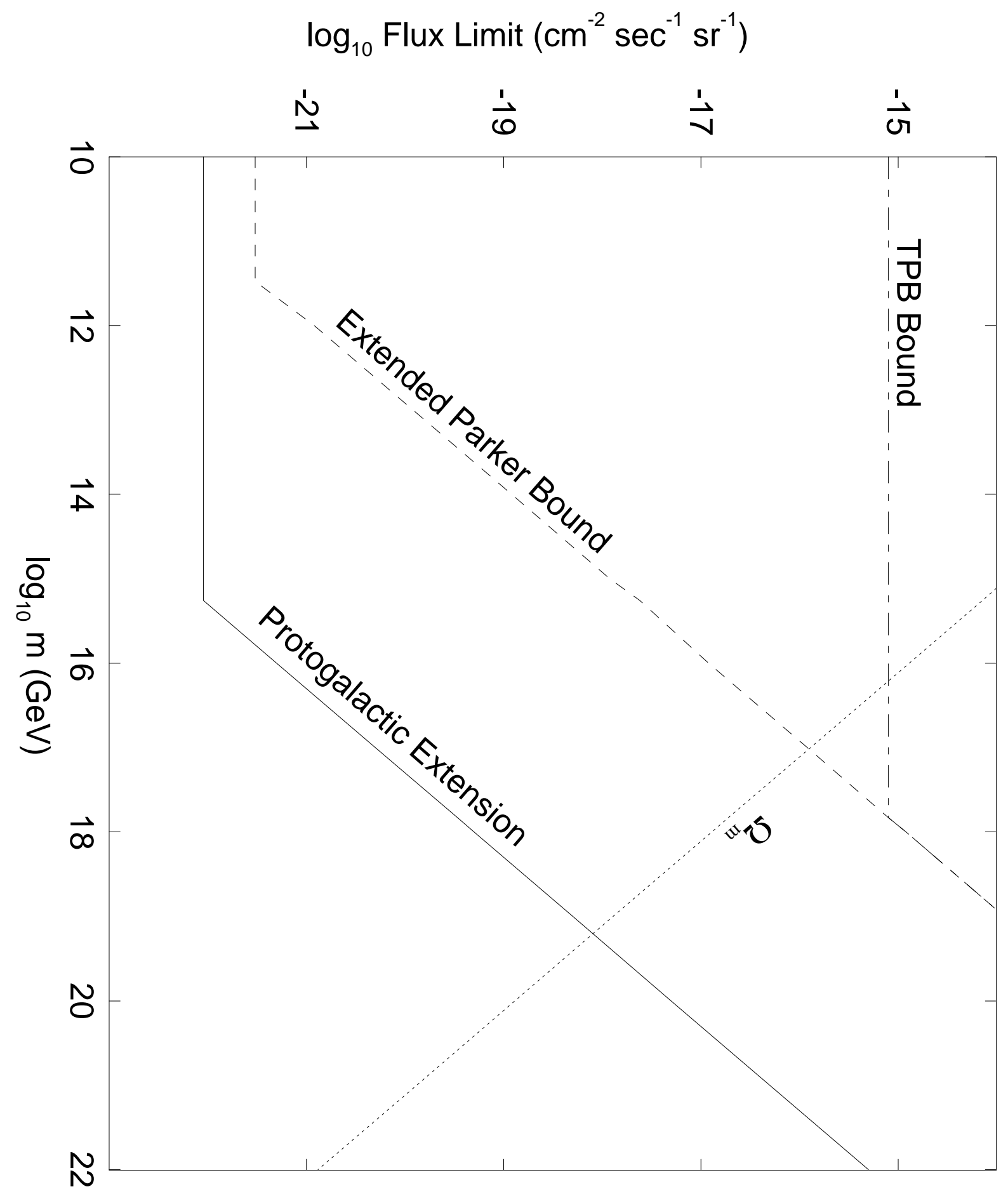

